\title{
Experiencing User-Centered Design (UCD) Practice (Case Study: Interactive Route Navigation Map of Bangkok Underground and Sky Train)
}

\author{
Waralak Vongdoiwang Siricharoen \\ Computer Science Department, School of Science, \\ 126/1, Vipavadee-rangsit Road, \\ University of the Thai Chamber of Commerce \\ Bangkok, Thailand \\ waralak_von@utcc.ac.th
}

\begin{abstract}
The research is aim to create MRT: Metropolitan Rapid Transit Authority of Thailand (Underground Train) and BTS: Bangkok Mass Transit System Public Company Limited (Sky Train) interactive route navigation map. This map shows the whole routes of both MRT and BTS train stations which are located in the interactive manner. The design is not only a proposal for showing the whole information about direction and price information of transportation by MRT and BTS in Bangkok. But also this research took these concepts one step further, by introducing interactive map which the viewers/passengers can access the information from everywhere via the internet. The goal of this interactive navigation map is to satisfy informative. Problem space includes 2 different user groups. Regardless of age or background, they do all have the same purpose; travel with the train. The User-Centered Design (UCD) methodology has been applied in developing processes of this research.
\end{abstract}

Keywords: User-Centered Design (UCD), Human Computer Interaction (HCI), Interactive, Map.

\section{Problem Statement and Motivation}

The BTS Sky train is the comfortable and convenient way to get around Bangkok. In service since 1999, it has transformed the face of public transportation in the Thai capital, for the first time offering both residents and visitors a comfortable ride through central Bangkok. The Bangkok's second major mass transit system (MRT Mass Rapid Transit: underground train) is on track for a test run on 2004 that reaches from the Northern train station of Bangsue to Hua Lumphong main railway station in a loop, connecting with the BTS on 3 different stations, namely: Silom, Sukhumvit, and Chatuchak Park ${ }^{1}$. MRT have a similar ticket system as BTS but both systems are for the time being not compatible. All stations of MRT can be reached via ramps and elevators, if needed, or alternatively via escalators.

\footnotetext{
${ }^{1}$ www.bts.com
} 
The finalized system of this research is web based version of MRT and BTS interactive route navigation map. The three main problems for the tourists/residents, who travel around Bangkok, are:

- The first problem is that MRT and BTS systems and services are still unknown to the tourists/residents; because the complete information of underground and sky train maps are not provided on the both MRT and BTS official web site. But there is some information that provided in web site of private organization for example, http://bangkok.sawadee.com/skytrain.htm, www.2bangkok.com, http://bangkok. sawadee.com/mrta.htm. As we can see information of MRT and BTS routes separately in Fig. 1 and 2.

- The second problem is that tourists/residents do not know the necessary details in order to using these transportation; such as, if where the train stations are, and how they connected along the train route or, how both BTS and MRT connected, How much does it cost for traveling from one station to another?. If we look at many interactive systems we can see that they have been designed by computer programmers and software engineers on the false assumption that everyone else in the world understands (or should understand) computers as well as they do. How can we make sure that interactive systems are better designed? [1]. Most of the problems we address in this course fall under the topic "how can we design it better?"

- The third problem is concerning the developing processes, the designers of the information system are actually very intelligent. Most designers of the system are computer experts and programmers: people familiar with computer systems, how they work and not really intimidated by them. In the mistaken that they are typical members of the public [1].

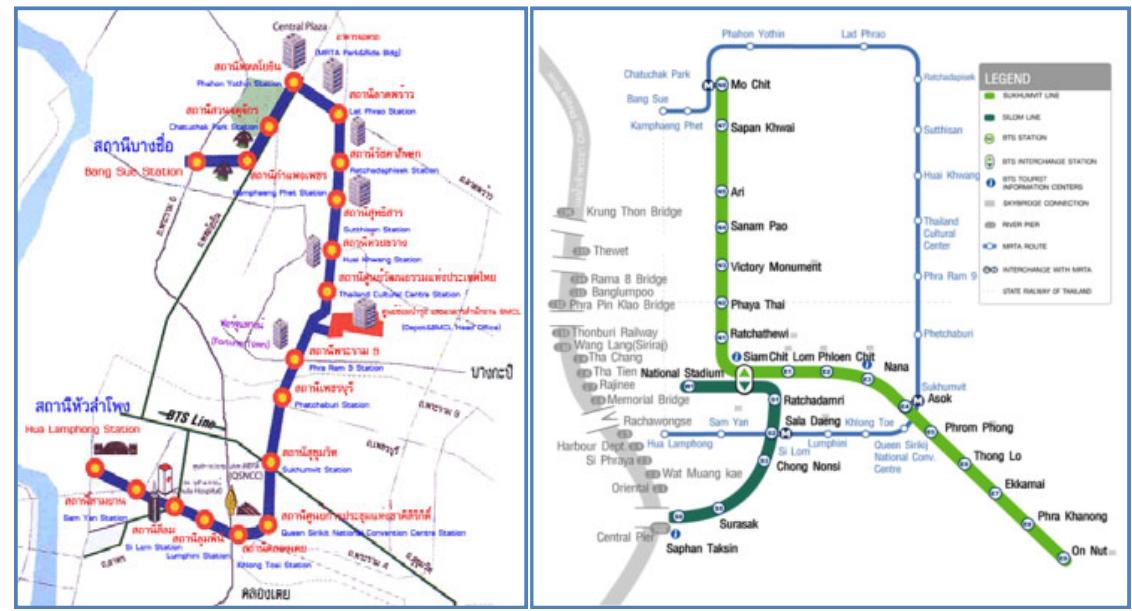

Fig. 1. MRT underground route map $^{2}$ (left) and BTS sky train route map (right)

\footnotetext{
${ }^{2}$ http://bangkok.sawadee.com
} 


\section{Research Objective Statements}

"Getting It Right the First Time"...is the most desirable thing that every developer wants. This research intends to apply the user-centered design methodology to create the interactive route navigation map. So the primary problem specification is to refer to any technological system which requires interaction with user, such as how can we design an interactive MRT and BTS map by integrating the user design center method, which is both convenient and informative?. Research Objectives is to help identify the primary needs the users will have off the train map, we have chosen to split the goals into usability goals, user experience goals, and, publication goals; as suggested [2].

- Usability Objectives: Because we assume a wide variety of users, it is important that the system is easy to use.

- User experience Objectives: First and foremost we wish the user to have a nice and easy experience using the public online interactive map. We try to imagine; a user on the station who looks at the interactive route pop up from inactive background and sees the clear train route. Maybe he can see the reality location of all the train stations. The users know at any given time where their closest station is located, our goal of making a service that is informative has been met.

- Publication Objectives: As the main industry in Thailand is tourism [3]; this research can be benefited to the tourists as well as residents. This work can provide the main transportation gateway of BTS and MRT in Bangkok. The first time visitor can use this web-based interactive map to see and learn how to travel around Bangkok using MRT and BTS.

\section{Interactive Manners and User-Centered Design (UCD)}

Interaction design aims to reduce the learning curve and to increase accuracy and efficiency of a task without diminishing usefulness. The objective is to reduce frustration and increase user productivity and satisfaction. Interaction design tries to understand certain users' requirements and then designing to gather and go above them by observing who wants to use it, and how they would like to use it. Relating users, designers gain the ability to better understand user goals and experiences. There are also positive side effects which include enhanced system capability awareness and user ownership or sometimes called participatory design. To enhance overall approval of the final product by adding a sense of ownership, because users who have been dynamic contributors in a system's development are more expected to feel that they are the owner of the product. The powerful, interactive systems will be relevant to its intended users, will support the tasks for which it is intended and make good use of technology. According to Ben Shneiderman, "The old computing is about what computers can do, the new computing is what users can do." [1]. Then any concern of the present and future of computing was restricted. Only especially skilled people could work with them. Ben Shneiderman also said in foreword (Sears and Jacko, 2003) that there are three essential topics to address in Human Computer Interaction (HCI). They are usable, universal, and useful. It is vitally important for system designers and HCI 
practitioners to distinguish between usefulness, usability and accessibility (universal) if they are to be as effective as possible in their work [1]: Useful means that the system supports user objectives, Usable means that it is supports these objectives in easyto-use ways, Accessible means that it can be used by the full range of intended users.

In order to accomplish these concepts, the UCD is the answer of how we come up with the system that can perform these essential concepts. Combining with a new methodology which is prototyping, the final result of prototyping studies would be directly convertible into efficient application code. On the usability evaluation side, the scenarios and prototypes would be readily converted into a simulation that would support human studies of effectiveness of the resultant designs [4]. The phrase interactive design refers to both the process and the results of design. User-centered design is itself an interactive process. If you believe in creating interaction between the system and the user, then it makes sense to employ the same concept of interaction between when the user is, in this case, the designer [4]. UCD was developed at the University of California at San Diego; the key features include [1]: 1. A central focus on the people who will use the systems, on their preferences and requirements, 2. Building simple models of the users, the tasks and the technological systems, 3. An iterative process and 4. Prototyping and the evaluation of alternatives by users."

The designer cannot passively go through a number of stages in linear manner, doing each stage once before signing it if and moving on like waterfall model. In UCD, by contrast, the designer and the design process must both work interactively. The emphases are design and evaluation as an interactive cycle. Designers cannot become stuck with their initial ideas and assumption, but must respond to data about what works and what does not. UCD forces designers to be explicit about decisions made, reviewing them through prototype and storyboards with the team and potential users. Designers need ways of sharing design thinking by producing physical representations of design ideas. These could be working computer-bases prototypes or as simple as a paper sketch [1]. It means that the design team and potential users can share in the process, evaluating the design ideas and suggesting improvements.

There is certainly the current high level of interest in multimedia interaction parts from up to date technical advance. While guidelines and research findings exist, so do large and significant gaps in knowledge. This is due to the inherent problem of predicting how system features will succeed or fail in realistic task setting. Moreover, iterative design and testing method are necessary [5]. One of the whole suggestive processes of UCD starts from Plan $\rightarrow$ Research $\rightarrow$ Design $\rightarrow$ Adapt $\rightarrow$ Measure [6]. UCD is an approach to design that grounds the process in information about the people who will use the product. UCD processes focus on users through the planning, design and development of a product [7]. There is an international standard that is the basis for many UCD methodologies. This standard (ISO 13407: Human-centered design process) [8] defines a general process for including human-centered activities throughout a development life-cycle. ISO 13407 provides guidance on achieving quality in use by incorporating user- centered design activities throughout the life cycle of interactive computer-based systems. The sequence in which these are performed and the level of effort and detail that is appropriate varies depending on the design environment and the stage of the design process [8]. 


\section{Case Study Walk through: The Development Processes}

In less well-defined projects, many designers have found real scenarios helpful to characterize that happen when users perform classic tasks. During the early design stages, data about current performance should be collected to provide a baseline. UCD is a flexible process for software development projects that enables teams to more effectively meet the needs of users and customers. In this version, the UCD activities are broken down into simple four phases: Analysis, Design, Implementation and Deployment, with suggested activities for each phase. They are as following:

\subsection{Analysis and Design}

\subsubsection{Finding the Objectives of the Research}

It should focus on usable, universal, and useful.

\subsubsection{Developers Brainstorming}

- Developers who have experience in coding for 5- 7 years, got together to discuss the experience on using the similar accessible system. One of the developers did not have any experience in using or creating the interactive map at all. Consequently they discussed how the system should work in their own perspective.

- The first problem occurred in this phase is that the requirements are not clear to developers. And as we set the methodology as UCD, then they must turn to users in order to get what users really need. They all have their own area of expertise such as some are very good in design, or coding, or graphic design.

- Learning from the other available systems, developers have the good design from these three accessible train interactive systems which are the Paris interactive map $^{3}$, the Seoul Metropolitan Rapid Transit interactive map ${ }^{4}$, and the Tokyo interactive map $^{5}$ as see in the Fig. 2.

Regardless of the method we choose to identify the appropriate tasks and requirements for your Web site, it is important that we conduct these activities before implementing any new design or major redesign of a site. This critical first step in the Web site design process will ensure that final design is user-friendly, flexible and adaptable, and will eliminate expensive and unnecessarily frequent redesigns.

Considering available interactive Thai interactive train map, they cannot show the whole route pop up from the screen and there is no information about the price from beginning to destination stations which are selected by the users. Also there is no information about connection of BTS and MRT route. Nevertheless the advantage of these existing interactive map Web sites is that they can show the surrounding area/tourism spots of each train stations.

\subsubsection{Finding What the Users Need}

Introduction an integral part of an effective user-centered design (UCD) by IBM; process is the gathering of requirements and the identification of common user tasks.

\footnotetext{
${ }^{3}$ http://www.ratp.info/orienter/cv/cv_en/carteparis.php

${ }^{4}$ http://www.smrt.co.kr/Train/Subwaymap/Eng/Subwaymap.jsp

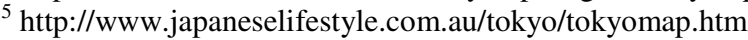


This part of the UCD process is just as important in Web site design and it is in software design. Interview user and focus group techniques had been applied in this stage. User questionnaires are familiar, inexpensive and generally acceptable companion for usability tests and expert reviews. The keys to successful questionnaires are clear goals in advance and development of focused items that help to attain those goals. Experienced questionnaire designer knows that care is also needed during design, administration, and data analysis [9].
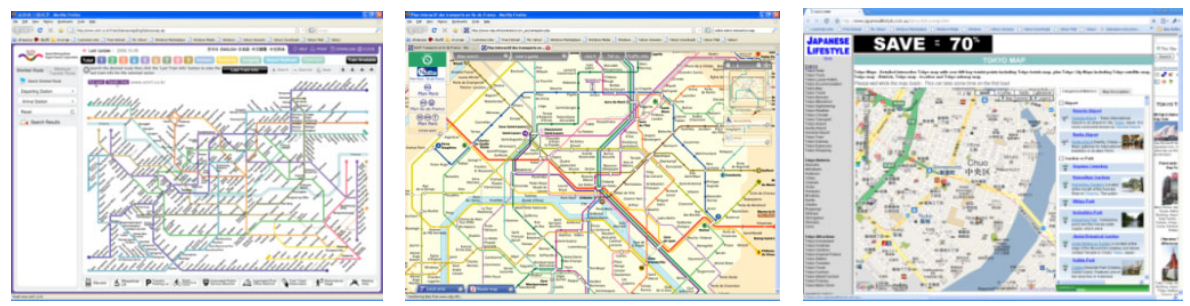

Fig. 2. Korea ${ }^{6}$, Paris $^{7}$, and Tokyo ${ }^{8}$ interactive map

As the users cannot imagine how the system work. Developers have to draw the few samples of system screen interface in order to show to the users. And then they discuss and choose the most preferable design or function that they want to be included in the screen. The reason that why we have to interview users before creating the prototype because we do not want to narrow the users' idea. This process might take very long time, because the users might not have any clue of what the system will look like, but we can discover what the important functions of the system is, and what kind of information the user needs. 10 open questions had been selected by developers and were distributed the 2 groups of the 40 users (age between $19-44$ years old). The first group is the users which are unfamiliar or little familiar to the BTS and MRT transportation; they do not often travel or never travel by BTS or MRT. The other group is the users which travel very frequently by BTS and MRT.

The results from the questionnaire conclusion (Fig. 3) show that the high similarity requirements are 1 . Route distinction (30\% and $29 \%), 2$. Price (18\% and 17\%), 3 . Travel time (11\% and 17\%), 4. Station name (16\% and 10\%), 5. Connection point of BTS and MRT (11\% and 6\%). Other suggestion topics are; color of each route, printable, zoom (in-out), bold font, bi-language (Thai-English).

\subsection{Implementation and Deployment}

After getting the system requirements; the developers pull out the similar users' needs from both 2 groups of users and make it dominant on prototype screens. The problem found in this stage is the coding problem. The first coding style, we applied PHP script to generate the database (it works as server-site script), the result system

\footnotetext{
${ }^{6} \mathrm{http}: / /$ www.smrt.co.kr/Train/Subwaymap/Eng/Subwaymap.jsp

${ }^{7}$ http://www.ratp.info/orienter/cv/cv_en/carteparis.php

${ }^{8} \mathrm{http}: / /$ www.ratp.info/orienter/cv/cv_en/carteparis.php
} 


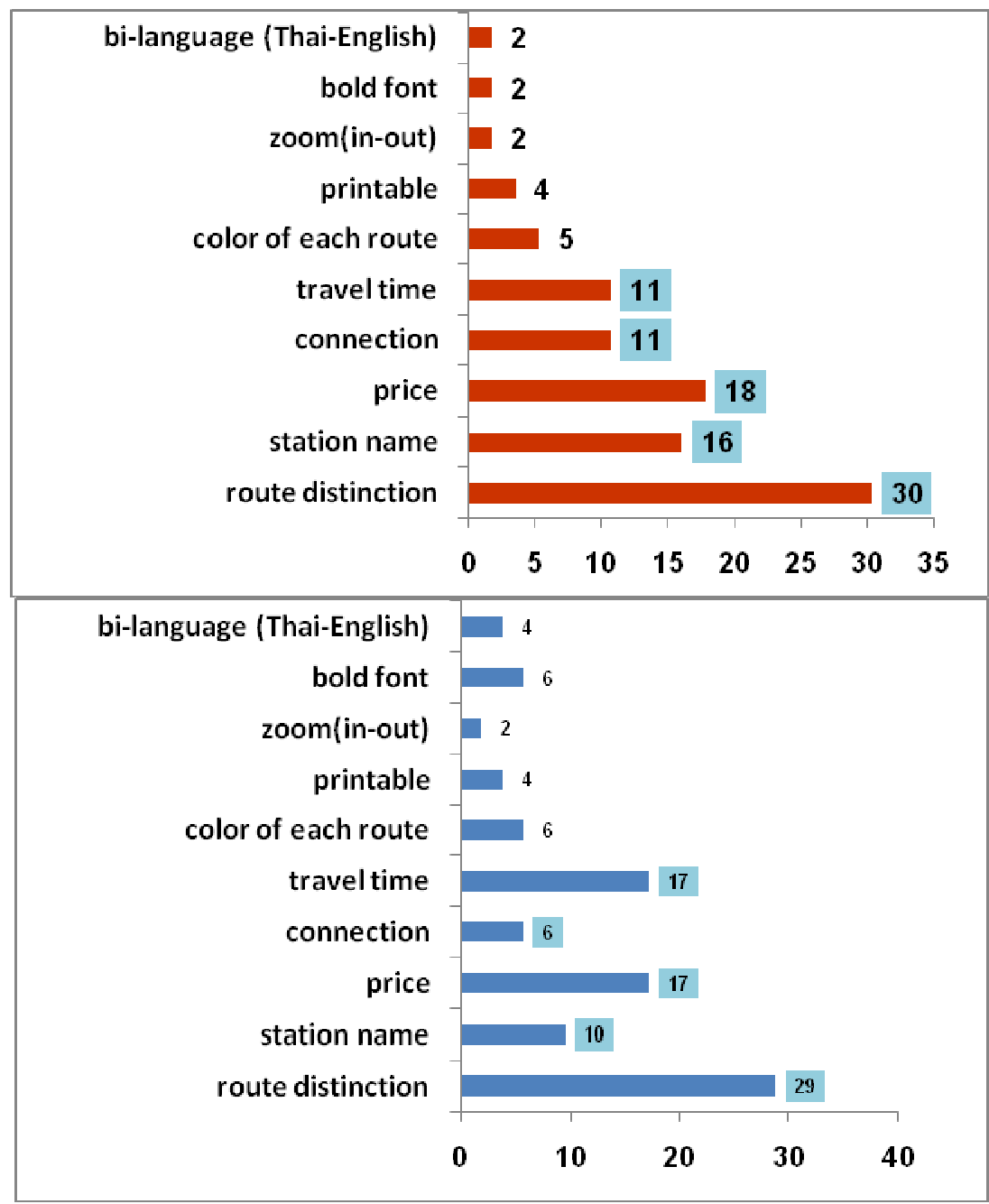

Fig. 3. Percentage comparison graph of suggestion topics between 2 groups of users: group1: unfamiliar user (above) group2: familiar user (below)

did not impress users, because it took a long time to show the train's route which user selected, even though the database is not so large. The developers decided to changecoding style from PHP to array instead. There are pros and cons here: Array style of coding took less time to process and made the system more efficient than PHP because it works on client site, regardless of the operating system, platform, speed of internet connection. However it might make difficulty in the future for developers to edit the code of the program. 

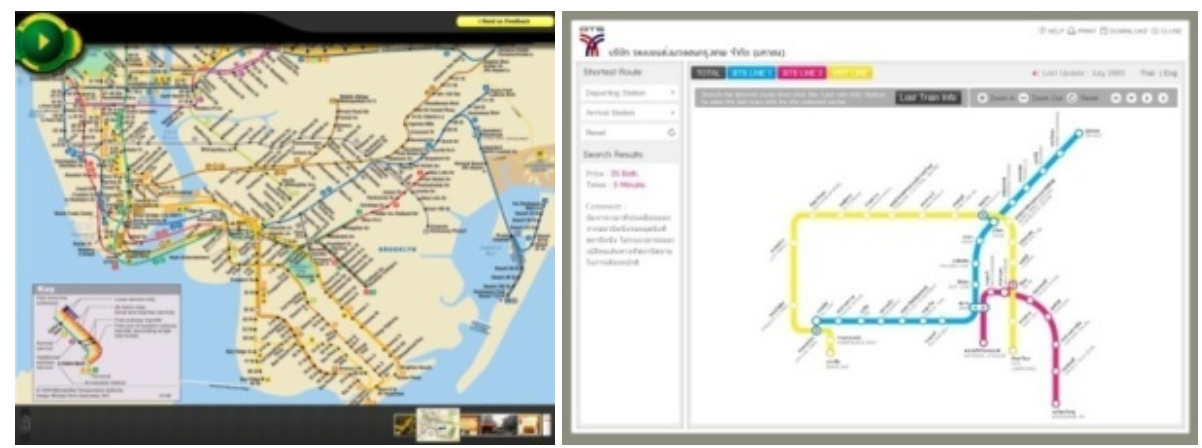

Fig. 4. The first interactive map which is not been selected (too much information on one page) (left) the first prototype screen with white background (right)
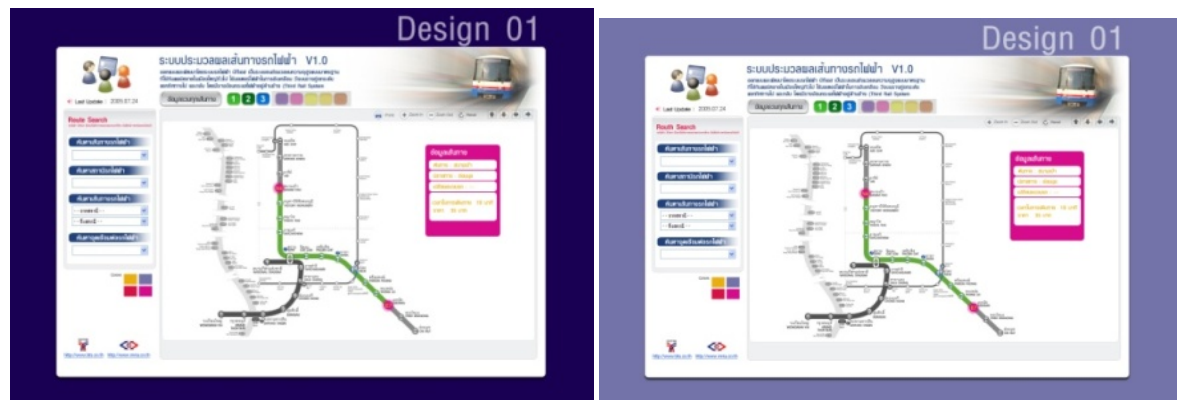

Fig. 5. The in-process screen design

The first draft design is based on the existing Bangkok interactive map, which contain too much information (Fig. 4 left). Accordingly, the developers considered the information. From the questionnaire and interview, the users do not need so many details, because they need simply 9-10 topics of content on the screen. They determined drop this design (Fig. 4 left). The next design is white background with the main train route distinction (Fig. 4 right), the users do not like the simple color, and they found them unattractive (Fig. 5 left). The developers show them to user then the user want the more colorful screen (primary color are yellow, red, and blue ${ }^{9}$ ) and then we select the blue color theme. ${ }^{10}$ Hence the next version draw on the dark blue background with a same blue shade of menu's color with the white background with the same train route distinction as same as the first design (Fig. 5 right). The users prefer this design but they want to make change to cooler tone of background color. And developers totally agree on this suggestion; the reason is that the interactive intend to provide the content in text and the user usually take time to look at the information

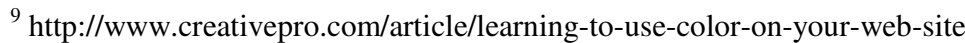

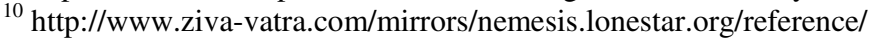
internet/web/color/charts/blue_01.html
} 
concern the route of the trains, as a result; in this case the user need comfort tone of color to their eyes for their eyes.

The last suggestion made by users is to construct the information universal use, which is to make web site available in both Thai and English language as see the last adjustment in Fig. 6 below.

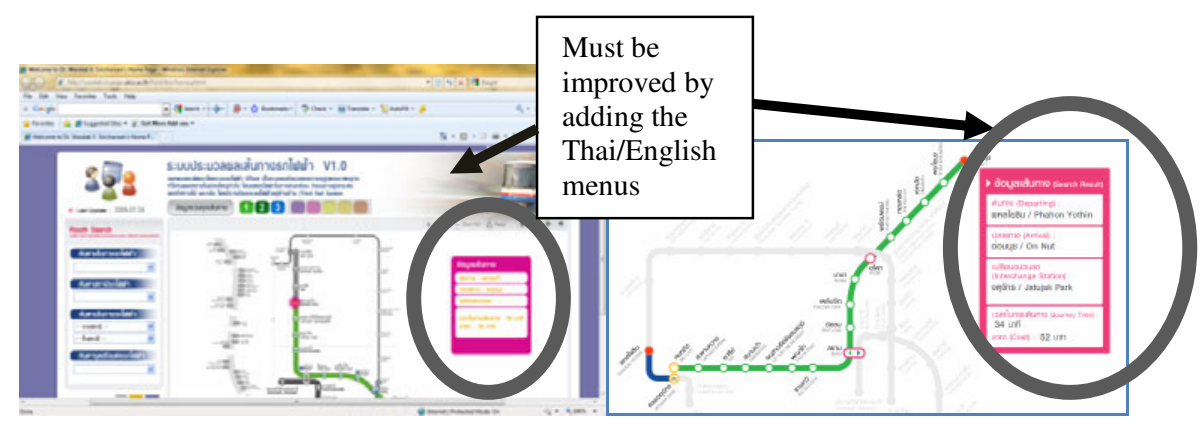

Fig. 6. The final web based interactive map

\section{Conclusions Recommendations}

User-centered design is not just about the user interface and the screen layout, colors, and buttons. It is much more, to come to a good user interface design at the end, it is essential to lay the foundation in the beginning. This foundation is an understanding of what end users really need and want "the user requirement". As already stated in the name of the process, "User-Centered" Design, the end-user should be involved in every step of software development processes and increase the ownership of the product. This investment of involving end users and customers early in the development process ensures that software products are produced that are demanded and accepted by users at the end. The recommendation further works are the links to tourist spots nearby station or link to the official web of BTS and MRT. There is the intention of the researcher to give this work to MRT and BTS and to make it available via their official web site. Now the final version is available at http://utcc2.utcc.ac.th/public_online/(Fig. 7.). Clearly after the last evaluation from the users, they say that the research work is very save time and money for the residents and tourists in order to prepare the trips before go to the train stations. The bottom line is UCD is the methodology which let the user get involve with the developing processes since the beginning of the project; there are advantage and disadvantage here. From the case study; the advantage are: increasing the acceptance of the product because of participatory and ownership in products, getting the real requirement from the users, and usable products. The disadvantages are: time consuming and costly because of iterative design, change of requirements, and argument between developers and users. 


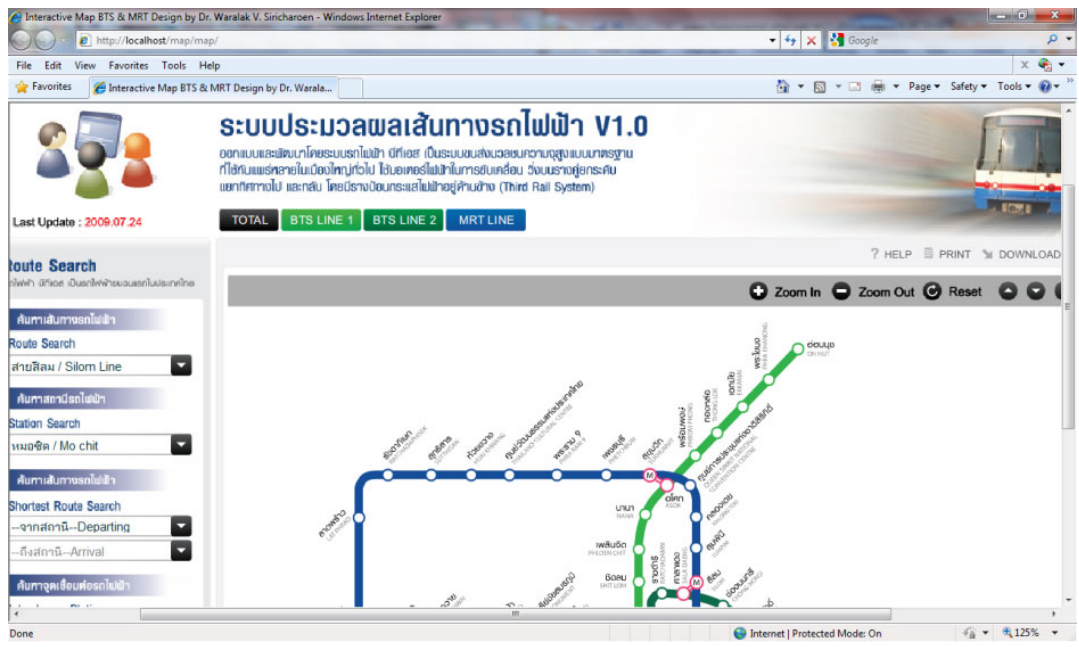

Fig. 7. The final web based interactive map

\section{References}

1. Smith-Atakan, S.: Human-Computer Interaction. In: Thomson Learning, ch. 1, 2, London, England (2006)

2. Sharp, H., Rogers, Y.: Preece J. Interaction Design: Beyond Human-Computer Interaction, 2nd edn. (2007), ISBN: 978-0-470-01866-8

3. iexplore.com. Where to go in Thailand (2009), http: / / www. iexplore.com/dmap/Thailand/Where+to+Go

4. Pew, R.W.: Evolution of Human-Computer Interaction: from Memex to Bluetooth and Beyond. In: The Human-Computer Interaction Handbook: Fundamentals, Evolving Technologies and Emerging Applications, p. 15 (2003)

5. Waterworth, J.A.: Multimedia interaction with Computers: Human Factor Issues. Ellis Horwood Series in Information Technology, West Sussex, England (1992)

6. Weissenberger, U., Thompson, C.F.: User-Centered Design SAP User Experience

7. Usability Professionals' Association: what is User-Centered Design? (2009), http: / / www . upassoc.org/usability_resources/about_usability/wh at_is_ucd.html

8. UsabilityNet : ISO 13407, Human centered design processes for interactive systems (2006), http://www. usabilitynet.org/tools/13407stds.htm

9. Shneiderman, B., Plaisant, C.: Designing the user interface: strategies for effective humancomputer interaction, 5th edn. Addison-Wesley, Boston (2010) 